\title{
molecules
}

ISSN 1420-3049

www.mdpi.com/journal/molecules

Article

\section{Headspace Solid-Phase Microextraction Analysis of Volatile Components in Narcissus tazetta var. chinensis Roem}

\section{Hsin-Chun Chen ${ }^{1, *}$, Hai-Shan $\mathrm{Chi}^{2}$ and Li-Yun Lin $^{3}$}

1 Department of Cosmeceutics, China Medical University, Taichung 404, Taiwan

2 Department of Horticultural Science and Graduate Institute of Horticulture, National Chiayi University, Chiayi 600, Taiwan; E-Mail: hschi@mail.ncyu.edu.tw

3 Department of Food Science and Technology, Hungkuang University, Taichung 433, Taiwan; E-Mail: 1ylin@sunrise.hk.edu.tw

* Author to whom correspondence should be addressed; E-Mail: d91628004@ntu.edu.tw; Tel.: +886-4-2205-3366 (ext. 5310); Fax: +886-4-2236-8557.

Received: 9 October 2013; in revised form: 28 October 2013 / Accepted: 1 November 2013 / Published: 6 November 2013

\begin{abstract}
The volatile components in single-flowered and double-flowered Chinese narcissus were identified by headspace-solid phase microextraction (HS-SPME) coupled with GC and GC/MS. Changes in aroma during the vase-life (days 0, 1, 2, 3, 4, 5 and 6) of two samples were also studied. A total of 35 compounds were identified, of which all were present in single-flowered and 26 in double-flowered samples. The main aroma components were $(E)$ - $\beta$-ocimene, and benzyl acetate. Single-flowered narcissus have a higher percentage of benzyl acetate, while double-flowered narcissus have a higher percentage of 1,8-cineole. In vase-life, the total volatile component content peaked on day 2 for single-flowered and day 3 for the double-flowered narcissus. For both single-flowered and double-flowered narcissus flowers, the total content of volatile components had decreased significantly by day 4 .
\end{abstract}

Keywords: Chinese narcissus; Narcissus tazetta var. chinensis Roem; HS-SPME; volatile compound 


\section{Introduction}

Chinese narcissus (Narcissus tazetta var. chinensis Roem) is a member of the Amaryllidaceae family and the Narcissus genus. It is a monocot plant whose flowers develop at high temperatures and bloom at lower temperatures. Featured during the Chinese Spring Festival, Chinese narcissus is a traditional and well-known Chinese flower with high economic and ornamental value [1]. It is a popular ornamental flower worldwide, especially in China and Taiwan, and narcissuses are widely cultivated in southern China [2]. Narcissus has a strong fragrance [3], that is highly valued in the fragrance industry [4].

Table 1. Comparisons of major volatile compounds reported in Narcissus flowers in published papers.

\begin{tabular}{|c|c|c|c|}
\hline Plant species/variety & Major compound & $\begin{array}{l}\text { Methods of } \\
\text { testing/extraction }\end{array}$ & Ref. \\
\hline Narcissus poeticus L. & $\begin{array}{l}\alpha \text {-terpineol, menthyl- }(E) \text {-isoeugenol, methyl- }(E)- \\
\text { isoeugenol, and benzyl benzoate }\end{array}$ & headspace & [3] \\
\hline N. trevithian and $N$. geranium & $\begin{array}{l}\text { Authors did not present any quantitative data on the } \\
\text { individual compounds. However, they reported } N \text {. } \\
\text { trevithian contains more phenolic compounds and fewer } \\
\text { esters compared to } N \text {. geranium }\end{array}$ & high vacuum distillation & {$[4]$} \\
\hline N. tazetta var. chinensis & $\begin{array}{l}\text { benzyl alcohol, } \alpha \text {-terpineol, } \gamma \text {-phenylpropyl alcohol, } 1,8- \\
\text { cineole, benzyl acetate, and linalool }\end{array}$ & hydrodistillation & {$[5]$} \\
\hline N. tazetta florepleno & $\begin{array}{l}\text { benzyl acetate, methyl anthranilate, benzyl alcohol, and } \\
\text { linalool }\end{array}$ & hydrodistillation & {$[6]$} \\
\hline Narcissus, two cultivars & benzyl acetate, benzyl alcohol, linalool, and indole & hydrodistillation & [7] \\
\hline Fresh narcissus flowers & benzyl alcohol, and $\alpha$-terpineol & headspace & {$[8]$} \\
\hline Narcissus flowers & $(E)-\beta$-ocimene and $\alpha$-terpineol & $\begin{array}{l}\text { Likens-Nickerson } \\
\text { extraction }\end{array}$ & {$[9]$} \\
\hline Narcissus flowers & $\begin{array}{l}\text { benzyl acetate, }(E) \text { - } \beta \text {-ocimene, } 3,4 \text {-dimethoxytoluene, } \\
\text { and } 3,5 \text {-dimethoxytoluene }\end{array}$ & headspace & {$[10]$} \\
\hline Zhangzhou narcissus flowers & $\begin{array}{l}\text { benzyl acetate, benzyl alcohol, indole, } \\
\text { 3,7-dimethyl-1,6-octadien-3-ol, and } \rho \text {-mentha-1,8-dien- } \\
\text { 4-yl acetate }\end{array}$ & hydrodistillation & {$[11]$} \\
\hline N. tazetta var. chinensis & benzyl acetate, linalool, and 1,8-cineole & hydrodistillation & {$[12]$} \\
\hline N. tazetta and & $\gamma$-terpinene for $N$. tazetta & lab-prepared absolutes & {$[13]$} \\
\hline N. tazetta subsp. tazetta & $\begin{array}{l}\gamma \text {-terpinene, linalool, and benzyl acetate for } N \text {. tazetta } \\
\text { subsp. tazetta }\end{array}$ & & \\
\hline N. taztta var. chinensis & benzyl acetate and $(E)-\beta$-ocimene & $\begin{array}{l}\text { headspace and } \\
\text { simultaneous } \\
\text { distillation }\end{array}$ & [14] \\
\hline$N$. tazetta and $N$. serotinus & $\begin{array}{l}\text { trans-ocimene for } N . \text { tazetta } \\
\text { benzyl acetate for } N . \text { serotinus. }\end{array}$ & hydrodistillation & {$[15]$} \\
\hline N. poeticus L. & $\begin{array}{l}\text { cinnamy alcohol, methyl isoeugenol, isoeugenol, methyl } \\
\text { eugenol, } \alpha \text {-terpinol, and phenyl propyl alcohol }\end{array}$ & $\begin{array}{l}\text { hexane and supercritical } \\
\mathrm{CO}_{2} \text { extraction }\end{array}$ & {$[16]$} \\
\hline N. pseudonarcissus L. & $(E)-\beta$-ocimene & headspace & [17] \\
\hline
\end{tabular}


Several studies on the volatile compounds of Narcissus spp. have been performed. Table 1 summarizes plant species/variety, method of testing/extraction, major volatile compounds and reference number in the published papers.

It was also recently confirmed that the use of headspace analysis provides a more natural profile that studies using hydrodistillation of plant volatiles [18]. SPME allows the sampling of the volatiles emitted by living plants in a fast and easy way [19], allowing the volatile compounds present in the headspace of odoriferous flowers in different flowering to be studied, including compounds that may not be detectable by conventional methods, such as solvent extractions, and hydrodistillation, but can be detected using HS-SPME [20].

Many previous studies have investigated the aroma of Chinese narcissus flowers, but few of them have discussed changes in narcissus flower aroma during the vase period. The aim of this work was to utilize HS-SPME method to investigate the volatile components and to understand the changes in these aromas during various stages of the vase period of fresh Chinese narcissus flowers.

\section{Results and Discussion}

\subsection{Analysis of the Volatiles in Fresh Single- and Double-Flowered Chinese Narcissus Flowers}

The volatile compounds in the narcissus flowers were analyzed by headspace solid-phase microextraction coupled with GC and with GC-MS. Tables 2, 3, and 4 show the 35 components that were identified in single-flowered samples, including 13 monoterpenes, three terpene alcohols, one terpene aldehyde, four terpene esters, one terpene oxide, one aromatic aldehyde, one aromatic alcohol, four aromatic esters, one sesquiterpene, three aliphatic esters, two hydrocarbons, and one other compound. A total of 26 components were identified in double-flowered samples, including 13 monoterpenes, three terpene alcohols, one terpene oxide, one aromatic alcohol, three aromatic esters, two aliphatic esters, two hydrocarbons, and one other compound. The main constituents of the Chinese narcissus flowers were $(E)$ - $\beta$-ocimene $(62.73 \%-66.06 \%)$, benzyl acetate $(11.65 \%-25.02 \%)$, (Z)- $\beta$-ocimene, 1,8-cineole, and linalool.

In a study by Sakai et al. [5], (Z)- $\beta$-ocimene was not detected. However, in a later study by Sakai [7], this component was detected. In a study by Surburg et al. [17], different headspace methods were used to analyze the aroma of Narcissus pseudonarcissus L. The main component was (E)- $\beta$-ocimene; a contribution of $75 \%$ was detected by the dynamic headspace method, while $30.1 \%$ was detected by the vacuum headspace method. In a study by Arai [14], the headspace method was used to analyze Narcissus tazetta var. chinensis, and a $51.19 \%$ contribution of $(E)$ - $\beta$-ocimene was detected. In this study, we used HS-SPME for analysis and found 62.73 and $66.06 \%$ contributions of $(E)$ - $\beta$-ocimene for the two samples used in this study. However, as shown in Table 1, no form of $\beta$-ocimene was reported as one of the major volatile components in distilled samples, therefore we postulate that heating during distillation decreased the content of this compound that could be detected. In addition, we found that single-flowered narcissus are rich in esters, including benzyl acetate, phenethyl acetate, isoamyl acetate, prenyl acetate, and 3-hexyl acetate. Among these ester components, the benzyl acetate content was the highest. Benzyl acetate was also a major component of narcissus flower aroma [3-7,10-14]. As shown in Table 1, benzyl acetate was reported as the first major volatile component in several 
studies, probably because of the low content of ocimene. In this study, the content of benzyl acetate was $25.02 \%$, next to $(E)-\beta$-ocimene $(67.02 \%)$. Volatile compound isolation methods by headspace or by distillation gave different data from this comparison. It was confirmed again that the use of headspace analysis provides a more natural profile than studies using hydrodistillation of plant volatiles [17].

In a study by Van Dort et al. [4] prenyl acetate was detected as a minor component. Regarding the terpene alcohols, linalool, and $\alpha$-terpineol were detected. Among these compounds, linalool is the major contributor to the aroma [5-7,10,12-14]. $\alpha$-Terpineol was reported as a major aromatic component of narcissus flowers in studies by Joulain [8], Loo and Richard [9], and Ehret et al. [3]. Indole is known as being very important in floral odors [4]. Although the indole content is low, its aroma threshold is low; thus, a pleasing and strong fragrance is emitted, even at low concentration. Fresh Chinese Narcissus flowers were picked and immediately analyzed and could therefore be considered as fresh at the time of SPME. As for aliphatic hydrocarbons, pentadecane was not detected by Sakai et al. [5] or Sakai [7], but this component was detected by Loo et al. [9]. The presence of $n$-alkanes, as a biomarker of fresh flowers, is ascribed to the Narcissus tazetta var. chinensis Roem flowers, and not contamination. Li et al. [20] and Shang et al. [21] also cited $n$-alkanes as a biomarker of living flowers for Michelia alba and Syring oblate flowers. In addition, sesquiterpene-type components are almost undetectable, which may be due to the poor absorption of sesquiterpene components by SPME [22,23]. To summarize, the main components of the floral scent of narcissus flowers were $(E)$ - $\beta$-ocimene, benzyl acetate, linalool, and indole. Among these components, benzyl acetate and $(E)$ - $\beta$-ocimene have floral aromas [24].

\subsection{Volatile Compounds over the Vase-Life of Narcissus tazetta var. chinensis Roem}

Table 3 shows the aroma constituents of Narcissus tazetta var. chinensis Roem at different flowering stages (flower buds, day 0; early flower blooming, day 1; flower blooming, day 2-3, late flower blooming, day 4; senescence, day 5-6 as analyzed by GC and GC/MS. All samples were placed in a plant tissue culture laboratory with a controllable environment, and the room temperature was set at $25{ }^{\circ} \mathrm{C}$. The vase life was 6 days. Every morning, the SPME method was used to extract aroma for analysis. The GC method was used for analysis and to compare the peak area content. As shown in Table 2, a total of 35 volatile compounds were identified for the single-flowered samples, for which the volatile components content peaked on day 2 , decreasing significantly by day 3 , and being lowest at day 6. Among these main components, $(E)$ - $\beta$-cimene, prenyl acetate, and benzyl acetate have the highest aroma content on day 2, with contents that decreased significantly thereafter. Among the monoterpene components, $\alpha$-pinene, sabinene, $\beta$-myrcene and $\gamma$-terpinene have the highest content on day 0 , decreasing with time thereafter. 6-Methyl-5-hepten-2-one was undetected on days 0-5, but it was detected in trace amounts on day 5 and peaked on day 6 . The odor of 6-methyl-5-hepten-2-one is metallic and wet-rubber-like as described by Chen et al. [24]. The compound was a speculated offodor of the Narcissus tazetta var. chinensis Roem. A total of 25 volatile compounds were identified for the double-flowered samples, and the content of their volatile components peaked on day 3 of the vase period. Among these major components, the aroma contents of $(Z)$ - $\beta$-ocimene, $(E)$ - $\beta$-ocimene, benzyl acetate, and linalool peaked on day 3 , decreasing significantly by day 4. Oyama-Okubo et al. [25] 
analyzed of the major scent compounds in cut flowers of 'Casa Blanca' lilies reported that total emissions of scent compounds peaked on the third day and then decreased.

Table 2. Volatile compounds of fresh flowers of Narcissus tazetta var. chinensis Roem.

\begin{tabular}{|c|c|c|c|c|}
\hline \multirow{2}{*}{ Compound } & \multirow{2}{*}{$\mathbf{R I}^{\mathbf{x}}$} & \multirow{2}{*}{$\mathbf{R I}^{\mathbf{y}}$} & \multicolumn{2}{|c|}{ Content $(\%)^{\mathrm{z}}$} \\
\hline & & & Single-flowered & Double-flowered \\
\hline \multicolumn{5}{|l|}{ Monoterpenes } \\
\hline$\alpha$-Pinene & 936 & 941 & $0.10 \pm 0.01$ & $0.31 \pm 0.05$ \\
\hline Sabinene & 973 & 967 & $0.01 \pm 0.00$ & $0.15 \pm 0.05$ \\
\hline$\beta$-Pinene & 978 & 971 & $0.02 \pm 0.01$ & $0.12 \pm 0.06$ \\
\hline Myrcene & 987 & 983 & $0.61 \pm 0.05$ & $1.22 \pm 0.09$ \\
\hline$\alpha$-Phellandrene & 1002 & 1002 & $<0.01$ & $<0.01$ \\
\hline$\delta$-3-Carene & 1010 & 1004 & $0.05 \pm 0.02$ & $0.40 \pm 0.15$ \\
\hline$\alpha$-Terpinene & 1013 & 1011 & $0.05 \pm 0.01$ & $0.08 \pm 0.01$ \\
\hline Limonene & 1025 & 1030 & $<0.01$ & $<0.01$ \\
\hline$(Z)$ - $\beta$-Ocimene & 1029 & 1037 & $1.80 \pm 0.49$ & $4.64 \pm 0.78$ \\
\hline (E)- $\beta$-Ocimene & 1041 & 1040 & $62.73 \pm 6.04$ & $66.06 \pm 11.01$ \\
\hline$\gamma$-Terpinene & 1051 & 1051 & $0.42 \pm 0.11$ & $0.27 \pm 0.08$ \\
\hline$\alpha$-Terpinolene & 1082 & 1085 & $0.04 \pm 0.01$ & $0.12 \pm 0.03$ \\
\hline allo-Ocimene & 1113 & 1116 & $1.21 \pm 0.07$ & $1.22 \pm 0.48$ \\
\hline \multicolumn{5}{|l|}{ Monoterpene alcohols } \\
\hline Linalool & 1086 & 1087 & $1.14 \pm 0.59$ & $1.71 \pm 0.35$ \\
\hline$\alpha$-Terpineol & 1176 & 1174 & $0.28 \pm 0.02$ & $0.06 \pm 0.02$ \\
\hline Myrtenol & 1178 & 1176 & $0.04 \pm 0.00$ & $0.04 \pm 0.01$ \\
\hline \multicolumn{5}{|l|}{ Monoterpene aldehyde } \\
\hline Citronellal & 1129 & 1129 & $<0.01$ & \\
\hline \multicolumn{5}{|l|}{ Monoterpene esters } \\
\hline Neryl acetate & 1342 & 1345 & $<0.01$ & \\
\hline Geranyl acetate & 1362 & 1362 & $<0.01$ & \\
\hline Methyl cinnamate & 1354 & 1373 & $0.10 \pm 0.01$ & \\
\hline Cinnamyl acetate & 1420 & 1422 & $0.02 \pm 0.01$ & \\
\hline \multicolumn{5}{|l|}{ Monoterpene oxide } \\
\hline 1,8 -Cineole & 1025 & 1025 & $1.49 \pm 0.50$ & $4.05 \pm 0.35$ \\
\hline Aromatic aldehyde & & & & \\
\hline Benzaldehyde & 964 & 964 & $<0.01$ & \\
\hline \multicolumn{5}{|l|}{ Aromatic alcohol } \\
\hline $\begin{array}{l}\text { Benzyl alcohol } \\
\text { Aromatic esters }\end{array}$ & 1006 & 1032 & $0.06 \pm 0.01$ & $0.01 \pm 0.00$ \\
\hline Benzyl acetate & 1134 & 1165 & $25.02 \pm 5.66$ & $11.65 \pm 4.22$ \\
\hline Phenethyl acetate & 1230 & 1269 & $1.12 \pm 0.16$ & $0.38 \pm 0.08$ \\
\hline 3-Phenylpropyl acetate & 1335 & 1357 & $0.07 \pm 0.03$ & $0.51 \pm 0.04$ \\
\hline $\begin{array}{l}\text { Benzyl benzoate } \\
\text { Sesquiterpene }\end{array}$ & 1730 & 1769 & $0.17 \pm 0.02$ & \\
\hline \multicolumn{5}{|l|}{ Aliphatic esters } \\
\hline Isoamyl acetate & 893 & 886 & $0.06 \pm 0.01$ & $0.03 \pm 0.01$ \\
\hline Prenyl acetate & 979 & 906 & $1.26 \pm 0.29$ & $2.18 \pm 0.38$ \\
\hline 3-Hexenyl acetate & 985 & 985 & $0.13 \pm 0.03$ & \\
\hline$\frac{\text { Hydrocarbons }}{\text { Pentadecane }}$ & 1500 & 1500 & $023+009$ & \\
\hline Eicosane & 2000 & 1983 & $0.08 \pm 0.03$ & $\begin{array}{l}0.02 \pm 0.01 \\
0.06 \pm 0.03\end{array}$ \\
\hline Other & & & $0.00 \pm 0.05$ & $0.00 \pm 0.05$ \\
\hline$\overline{\text { Indole }}$ & 1257 & 1295 & $0.33 \pm 0.11$ & $0.26 \pm 0.04$ \\
\hline
\end{tabular}

${ }^{\mathrm{x}}$ Literature Retention indices obtained from [26-28] and reference were checked for all compounds on DB-1 column; ${ }^{\mathrm{y}}$ Retention indices obtained using series of $n$-alkanes $\left(\mathrm{C}_{5}-\mathrm{C}_{25}\right)$ on DB-1 column; ${ }^{\mathrm{z}}$ Values are means $\pm \mathrm{SD}$ of six replicates. 
Table 3. Volatile compounds on vase life of Narcissus tazetta var. chinensis Roem.

\begin{tabular}{|c|c|c|c|c|c|c|c|c|c|c|c|c|c|c|c|}
\hline \multirow{2}{*}{ Compound } & \multirow{2}{*}{$\mathbf{R I}^{\mathrm{x}}$} & \multicolumn{7}{|c|}{ Single-flowered (peak areas) ${ }^{\mathrm{y}}$} & \multicolumn{7}{|c|}{ Double-flowered (peak areas) } \\
\hline & & $\mathbf{0}$ & 1 & 2 & 3 & 4 & 5 & 6 & $\mathbf{0}$ & 1 & 2 & 3 & 4 & 5 & 6 \\
\hline \multicolumn{16}{|l|}{ Monoterpenes } \\
\hline$\alpha$-Pinene & 941 & $8.99 b c$ & $4.82 \mathrm{~d}$ & $4.28 \mathrm{de}$ & $2.01 \mathrm{e}$ & & & & $11.28 \mathrm{bc}$ & $13.23 b$ & $17.30 \mathrm{a}$ & $18.19 \mathrm{a}$ & $9.65 \mathrm{bc}$ & $9.50 \mathrm{bc}$ & \\
\hline Sabinene & 967 & $1.30 \mathrm{~d}$ & $0.69 \mathrm{e}$ & $0.10 \mathrm{f}$ & & & & & $5.17 \mathrm{a}$ & $1.82 \mathrm{c}$ & $2.61 b$ & $1.70 \mathrm{c}$ & & & \\
\hline$\beta$-Pinene & 976 & $1.21 \mathrm{c}$ & $0.84 \mathrm{c}$ & $0.73 \mathrm{c}$ & & & & & $3.54 \mathrm{~b}$ & $2.03 \mathrm{c}$ & $9.48 \mathrm{a}$ & $1.87 \mathrm{c}$ & & & \\
\hline Myrcene & 983 & $30.88 \mathrm{~d}$ & $22.61 \mathrm{e}$ & $25.15 d$ & $8.87 \mathrm{fg}$ & $1.81 \mathrm{~g}$ & & & $29.16 \mathrm{~d}$ & $46.25 c$ & $68.39 b$ & $107.22 \mathrm{a}$ & $25.84 d$ & $14.64 \mathrm{ef}$ & $13.34 \mathrm{f}$ \\
\hline$\alpha$-Phellandrene & 1002 & & & $<0.10$ & & & & & & & & & & & \\
\hline$\delta$-3-Carene & 1004 & $2.43 \mathrm{f}$ & $1.91 \mathrm{f}$ & $1.90 \mathrm{f}$ & $1.32 \mathrm{f}$ & $1.11 \mathrm{f}$ & & & $10.19 \mathrm{e}$ & $20.35 b$ & $36.72 \mathrm{a}$ & $21.80 \mathrm{~b}$ & $18.30 \mathrm{c}$ & $17.10 \mathrm{c}$ & $13.30 \mathrm{~d}$ \\
\hline$\alpha$-Terpinene & 1011 & $1.00 \mathrm{cde}$ & $1.99 \mathrm{~b}$ & $2.74 \mathrm{a}$ & & & & & $1.23 \mathrm{~cd}$ & $0.91 \mathrm{e}$ & $0.89 \mathrm{e}$ & $1.26 \mathrm{c}$ & $1.27 \mathrm{c}$ & $0.98 \mathrm{de}$ & $0.42 \mathrm{f}$ \\
\hline Limonene & 1030 & & & $<0.10$ & & & & & & & & & & & \\
\hline$(Z)$ - $\beta$-ocimene & 1037 & $43.81 \mathrm{de}$ & $139.76 \mathrm{c}$ & $144.44 \mathrm{c}$ & $64.98 \mathrm{~d}$ & $9.12 \mathrm{fg}$ & $2.81 \mathrm{~g}$ & $1.83 \mathrm{~g}$ & $28.70 \mathrm{efg}$ & $137.02 \mathrm{c}$ & $221.85 b$ & $698.81 \mathrm{a}$ & $237.87 b$ & $72.20 \mathrm{~d}$ & $35.13 \mathrm{ef}$ \\
\hline$(E)$ - $\beta$-ocimene & 1040 & $792.65 \mathrm{ef}$ & $2271.78 d$ & $2299.77 d$ & $645.21 \mathrm{f}$ & $27.79 \mathrm{~h}$ & $4.64 h$ & $2.24 \mathrm{~h}$ & $311.77 \mathrm{~g}$ & $2694.95 \mathrm{c}$ & $3147.59 b$ & $9914.71 \mathrm{a}$ & $3371.10 b$ & $1024.45 \mathrm{e}$ & $659.79 f$ \\
\hline$\gamma$-Terpinene & 1051 & $18.09 \mathrm{c}$ & $9.50 \mathrm{~d}$ & $7.47 \mathrm{~d}$ & $4.20 \mathrm{e}$ & $3.89 \mathrm{e}$ & $1.48 \mathrm{e}$ & $1.39 \mathrm{e}$ & $8.72 \mathrm{~d}$ & $16.68 \mathrm{c}$ & $18.88 \mathrm{bc}$ & $21.77 \mathrm{ab}$ & $22.62 \mathrm{a}$ & $21.67 \mathrm{ab}$ & $18.75 \mathrm{c}$ \\
\hline$\alpha$-Terpinolene & 1085 & $2.41 \mathrm{e}$ & $2.01 \mathrm{e}$ & $1.76 \mathrm{f}$ & & & & & $2.24 \mathrm{e}$ & $3.23 \mathrm{~d}$ & $4.33 \mathrm{c}$ & $8.31 \mathrm{a}$ & $5.83 b$ & & \\
\hline Allo-ocimene & 1116 & $37.71 \mathrm{f}$ & $44.83 \mathrm{e}$ & $49.57 \mathrm{~d}$ & $15.48 \mathrm{~h}$ & $1.28 \mathrm{j}$ & & & $7.87 \mathrm{i}$ & $48.03 \mathrm{de}$ & $58.36 \mathrm{c}$ & $184.16 \mathrm{a}$ & $70.96 b$ & $26.29 \mathrm{~g}$ & $16.65 \mathrm{~h}$ \\
\hline \multicolumn{16}{|c|}{ Monoterpene alcohols } \\
\hline Linalool & 1087 & $58.85 \mathrm{~g}$ & $112.42 \mathrm{f}$ & $109.84 \mathrm{f}$ & $51.48 \mathrm{~g}$ & $20.99 \mathrm{i}$ & $17.78 \mathrm{i}$ & $1.21 \mathrm{j}$ & $21.43 \mathrm{i}$ & $147.11 \mathrm{de}$ & $188.91 \mathrm{c}$ & $280.72 \mathrm{a}$ & $243.05 b$ & $159.81 d$ & $32.88 \mathrm{~h}$ \\
\hline$\alpha$-Terpineol & 1174 & $12.11 \mathrm{~b}$ & $15.21 \mathrm{a}$ & $3.61 \mathrm{c}$ & $3.06 \mathrm{c}$ & & & & & & & & & & \\
\hline Myrtenol & 1176 & $1.34 \mathrm{~g}$ & $1.80 \mathrm{~d}$ & $2.03 b$ & $1.45 \mathrm{f}$ & & & & & $1.62 \mathrm{e}$ & $1.95 b c$ & $3.48 \mathrm{a}$ & $1.90 \mathrm{c}$ & & \\
\hline \multicolumn{16}{|l|}{ Monoterpene ketone } \\
\hline $\begin{array}{l}\text { 6-Methyl-5- } \\
\text { hepten-2-one }\end{array}$ & 965 & & & & & & $0.10 \mathrm{~b}$ & $11.10 \mathrm{a}$ & & & & & & & \\
\hline \multicolumn{16}{|l|}{ Monoterpene esters } \\
\hline Geranyl acetate & 1362 & & $4.49 \mathrm{a}$ & $3.01 \mathrm{~b}$ & $2.10 \mathrm{c}$ & & & & & & & & & & \\
\hline Methyl cinnamate & 1373 & & $3.70 \mathrm{e}$ & $3.76 \mathrm{de}$ & $4.71 \mathrm{~cd}$ & $4.43 \mathrm{cde}$ & $5.23 \mathrm{c}$ & & & $4.22 \mathrm{de}$ & $3.72 \mathrm{de}$ & $11.06 \mathrm{a}$ & $9.56 b$ & & \\
\hline Cinnamyl acetate & 1422 & & & & & & $3.20 \mathrm{c}$ & & $6.08 \mathrm{a}$ & $4.86 \mathrm{~b}$ & & & & & \\
\hline \multicolumn{16}{|l|}{ Monoterpene oxide } \\
\hline 1,8-Cineole & 1025 & $143.23 \mathrm{~d}$ & $158.73 \mathrm{~d}$ & $144.65 \mathrm{~d}$ & $64.14 \mathrm{~g}$ & $11.56 \mathrm{~h}$ & $2.76 \mathrm{~h}$ & $1.45 \mathrm{~h}$ & $85.13 \mathrm{f}$ & $222.04 \mathrm{c}$ & $268.56 b$ & $450.91 \mathrm{a}$ & $271.20 \mathrm{~b}$ & $122.62 \mathrm{e}$ & $65.85 \mathrm{fg}$ \\
\hline
\end{tabular}


Table 3. Cont.

\begin{tabular}{|c|c|c|c|c|c|c|c|c|c|c|c|c|c|c|c|}
\hline \multirow{2}{*}{ Compound } & \multirow{2}{*}{$\mathbf{R I}^{\mathbf{x}}$} & \multicolumn{7}{|c|}{ Single-flowered (peak areas) ${ }^{y}$} & \multicolumn{7}{|c|}{ Double-flowered (peak areas) } \\
\hline & & $\mathbf{0}$ & 1 & 2 & 3 & 4 & 5 & 6 & $\mathbf{0}$ & 1 & 2 & 3 & 4 & 5 & 6 \\
\hline \multicolumn{16}{|l|}{$\underline{\text { Aromatic alcohol }}$} \\
\hline Benzyl alcohol & 1032 & $1.40 \mathrm{~b}$ & $1.82 \mathrm{a}$ & $1.51 \mathrm{~b}$ & & & & & & & $0.72 \mathrm{~d}$ & $1.12 \mathrm{c}$ & & & \\
\hline \multicolumn{16}{|l|}{ Aromatic esters } \\
\hline Benzyl acetate & 1165 & $675.59 \mathrm{e}$ & $1324.56 b$ & $1390.43 b$ & 119.50hi & $20.52 \mathrm{j}$ & $7.10 \mathrm{j}$ & $4.47 \mathrm{j}$ & $186.44 \mathrm{gh}$ & $927.25 \mathrm{~d}$ & $1023.59 \mathrm{c}$ & $1598.03 \mathrm{a}$ & $568.64 f$ & $256.70 \mathrm{~g}$ & $99.44 \mathrm{i}$ \\
\hline Phenethyl acetate & 1169 & $15.59 \mathrm{~g}$ & $24.91 \mathrm{e}$ & $18.12 \mathrm{fg}$ & $6.90 \mathrm{~h}$ & $2.78 \mathrm{i}$ & $1.78 \mathrm{i}$ & & $46.14 b$ & $32.15 d$ & $20.86 f$ & $53.12 \mathrm{a}$ & $38.77 \mathrm{c}$ & $14.91 \mathrm{~g}$ & $7.81 \mathrm{~h}$ \\
\hline 3-Phenylpropyl acetate & 1357 & & & & & & & & & $6.43 \mathrm{~d}$ & $12.02 \mathrm{c}$ & $63.45 \mathrm{a}$ & $34.34 \mathrm{~b}$ & $6.37 \mathrm{~d}$ & \\
\hline Benzyl benzoate & 1769 & & & & $5.31 \mathrm{a}$ & $3.79 b$ & $1.79 \mathrm{c}$ & & & & & & & & \\
\hline \multicolumn{16}{|l|}{ Sesquiterpene } \\
\hline$\beta$-caryophyllene & 1430 & & $8.15 \mathrm{a}$ & $2.56 \mathrm{c}$ & $2.50 \mathrm{c}$ & $4.32 b$ & $4.22 b$ & & & & & & & & \\
\hline \multicolumn{16}{|l|}{ Aliphatic esters } \\
\hline Isoamyl acetate & 886 & $2.41 \mathrm{~g}$ & $2.94 \mathrm{~g}$ & $3.10 \mathrm{~g}$ & $11.15 b c$ & $8.13 d$ & $11.98 \mathrm{~b}$ & $6.10 \mathrm{e}$ & $3.69 \mathrm{~g}$ & $4.15 f g$ & $5.80 \mathrm{ef}$ & $5.85 \mathrm{ef}$ & $9.64 \mathrm{~cd}$ & $17.54 \mathrm{a}$ & $17.59 \mathrm{a}$ \\
\hline Prenyl acetate & 906 & $50.71 \mathrm{e}$ & $62.61 d$ & $65.91 \mathrm{~d}$ & $40.82 f$ & $3.27 \mathrm{~h}$ & $2.05 \mathrm{~h}$ & & $24.15 \mathrm{~g}$ & $108.26 \mathrm{c}$ & $147.73 a$ & $125.11 b$ & $123.04 b$ & $44.73 \mathrm{f}$ & $21.61 \mathrm{~g}$ \\
\hline 3-Hexenyl acetate & 985 & & & & & $1.03 b$ & $2.79 \mathrm{a}$ & & & & & & & & \\
\hline \multicolumn{16}{|l|}{ Hydrocarbons } \\
\hline Pentadecane & 1500 & $6.80 \mathrm{e}$ & $11.61 \mathrm{de}$ & $15.22 \mathrm{~d}$ & $18.73 \mathrm{~d}$ & $15.70 \mathrm{~d}$ & $15.00 \mathrm{~d}$ & $5.51 \mathrm{e}$ & $50.10 \mathrm{a}$ & $43.63 \mathrm{abc}$ & $37.21 \mathrm{c}$ & $47.12 \mathrm{ab}$ & $42.67 \mathrm{bc}$ & & \\
\hline Eicosane & 1983 & $7.87 \mathrm{~b}$ & $4.31 \mathrm{~d}$ & $3.49 \mathrm{e}$ & $2.84 \mathrm{f}$ & & & & & $5.03 \mathrm{c}$ & $4.02 \mathrm{~d}$ & $11.61 \mathrm{a}$ & $5.11 \mathrm{c}$ & $2.08 \mathrm{~g}$ & \\
\hline \multicolumn{16}{|l|}{ Other } \\
\hline Indole & 1295 & $12.50 \mathrm{ef}$ & $36.07 \mathrm{~b}$ & $34.48 \mathrm{~b}$ & $18.03 \mathrm{a}$ & $1.81 \mathrm{~h}$ & $0.61 \mathrm{~h}$ & & & $30.26 \mathrm{c}$ & $41.90 \mathrm{a}$ & $19.40 \mathrm{~d}$ & $14.22 \mathrm{e}$ & $10.52 \mathrm{fg}$ & $9.35 \mathrm{~g}$ \\
\hline
\end{tabular}

${ }^{\mathrm{x}}$ Retention indices, using paraffin $\left(\mathrm{C}_{5}-\mathrm{C}_{25}\right)$ as references; ${ }^{\mathrm{y}}$ Values are means of six replicates. Values having different superscripts are significantly $(p<0.05)$ different. 
Table 4. Peak areas of chemical groups of volatile compounds on vase life of Narcissus tazetta var. chinensis Roem.

\begin{tabular}{|c|c|c|c|c|c|c|c|c|c|c|c|c|c|c|}
\hline \multirow{2}{*}{ Compounds } & \multicolumn{7}{|c|}{ Single-flowered (peak areas) } & \multicolumn{7}{|c|}{ Double-flowered (peak areas) } \\
\hline & $\mathbf{0}$ & 1 & 2 & 3 & 4 & 5 & 6 & $\mathbf{0}$ & 1 & 2 & 3 & 4 & 5 & 6 \\
\hline Monoterpenes & 940.48 & 2500.74 & 2537.91 & 742.07 & 45.00 & 8.93 & 5.46 & 419.87 & 2984.5 & 3586.4 & 10979.8 & 3763.44 & 1186.83 & 757.38 \\
\hline Monoterpene alcohols & 72.30 & 129.43 & 115.48 & 55.99 & 20.99 & 17.78 & 1.21 & 21.43 & 148.73 & 190.86 & 284.2 & 244.95 & 159.81 & 32.88 \\
\hline Monoterpene ketone & & & & & & 0.10 & 11.10 & & & & & & & \\
\hline Monoterpene esters & & 8.19 & 6.77 & 6.81 & 4.43 & 8.43 & & 6.08 & 9.08 & 3.72 & 11.06 & 9.56 & & \\
\hline Monoterpene oxide & 143.23 & 158.73 & 144.65 & 64.14 & 11.56 & 2.76 & 1.45 & 85.13 & 222.04 & 268.56 & 450.91 & 271.2 & 122.62 & 65.85 \\
\hline Aromatic alcohols & 1.40 & 1.82 & 1.51 & & & & & & & 0.72 & 1.12 & & & \\
\hline Aromatic esters & 691.18 & 1349.47 & 1408.55 & 131.71 & 27.09 & 10.67 & 4.47 & 232.58 & 965.83 & 1056.47 & 1714.60 & 641.75 & 277.98 & 107.25 \\
\hline Sesquiterpene & & 8.15 & 2.56 & 2.50 & 4.32 & 4.22 & & & & & & & & \\
\hline Aliphatic esters & 53.12 & 65.55 & 69.01 & 51.97 & 12.43 & 16.82 & 6.10 & 27.84 & 112.41 & 153.53 & 130.96 & 132.68 & 62.27 & 39.20 \\
\hline Hydrocarbons & 14.67 & 15.92 & 18.71 & 21.57 & 15.70 & 15.00 & 5.51 & 50.10 & 48.66 & 41.23 & 58.73 & 47.78 & 2.08 & \\
\hline Other & 12.50 & 36.07 & 34.48 & 18.03 & 1.81 & 0.61 & & & 30.26 & 41.90 & 19.40 & 14.22 & 10.52 & 9.35 \\
\hline
\end{tabular}


For both single-flowered and double-flowered narcissus flowers, the total content of volatile components had decreased significantly by day 4 , and the total volatile component was lowest on day 6 .

\subsection{Comparison of Volatile Compounds from Single-Flowered and Double-Flowered of Narcissus tazetta var. chinensis Roem}

Due to their different relative contents, the two narcissus flower types might have different scents. More ester compounds were identified in single-flowered samples than in double-flowered samples, including benzyl benzoate, and 3-hexenyl acetate, which may contribute to the aroma profile of the flowers. As shown in Table 4, Single-flowered samples have lower total peak areas and higher percentages of sesquiterpenes than the double-flowered samples.

\section{Experimental}

\subsection{Plant Materials}

Single-flowered and double-flowered narcissus bulbs were purchased from Zhangzhou, Fujian Province, China. These bulbs were placed in pots containing water and then left under outdoor light. The bulbs were incubated for 35 days until they blossomed.

\subsection{Methods}

\subsubsection{Volatile Components of Narcissus Flowers}

(1) Aroma components of the single- and double-flowered Chinese narcissus flowers: Fresh singleand double-flowered narcissus flowers in full bloom (ten each) were picked and immediately placed into sealed bottle. The SPME method was used to extract the aroma components. This experiment and all other experiments in this study were performed with six replicates.

(2) Volatile compounds during the vase-life of Narcissus tazetta var. chinensis Roem: Samples of narcissus bulbs about to flower were placed in a tissue culture laboratory controlled at $25{ }^{\circ} \mathrm{C}$. The samples were exposed to $12 \mathrm{~h}$ of light and $12 \mathrm{~h}$ of darkness every day. Fresh budding single-flowered and double-flowered narcissus flowers (one flower each) were selected on a day then defined as day 0. These two samples were cut and inserted into two water-containing test bottle (precleaned \# 27343 22-mL clear screw cap vials; Supelco, Bellefonte, PA, USA). From day 0 to day 6 at 10:00-12:00 in the morning, the SPME method was used to extract aroma to monitor the changes thereof.

\subsubsection{Analysis of Volatile Compounds}

(1) HS-SPME analysis. A 50/30- $\mu \mathrm{m}$ divinylbenzene/carboxen/polydimethylsiloxane fiber (Supelco, Inc.) was used for aroma extraction. The SPME fiber was exposed to each sample for $30 \mathrm{~min}$ at $25^{\circ} \mathrm{C}$, after which each sample was injected into a gas chromatograph injection unit. Peak area data reported from the integrator was used for quantification.

(2) Analysis of volatile components of samples by GC: Qualitative and quantitative analyses of the volatile compounds were conducted using an Agilent $6890 \mathrm{GC}$ equipped with a $60 \mathrm{~m} \times 0.25 \mathrm{~mm}$ i.d. DB-1 fused-silica capillary column with a film thickness of $0.25 \mu \mathrm{m}$ and a flame ionization detector. 
The injector and detector temperatures were maintained at $250{ }^{\circ} \mathrm{C}$ and $300{ }^{\circ} \mathrm{C}$, respectively. The oven temperature was held at $40{ }^{\circ} \mathrm{C}$ for $1 \mathrm{~min}$ and then raised to $200{ }^{\circ} \mathrm{C}$ at $2{ }^{\circ} \mathrm{C} / \mathrm{min}$ and held for $9 \mathrm{~min}$. The carrier gas (nitrogen) flow rate was $1 \mathrm{~mL} / \mathrm{min}$. Kovats indices were calculated for the separated components relative to a $\mathrm{C}_{5}-\mathrm{C}_{25} \mathrm{n}$-alkanes mixture [29].

(3) Analysis of volatile components of samples by GC-MS: The volatile compounds were identified using an Agilent $6890 \mathrm{GC}$ equipped with a $60 \mathrm{~m} \times 0.25 \mathrm{~mm}$ i.d. DB-1 fused-silica capillary column with a film thickness of $0.25 \mu \mathrm{m}$ coupled to an Agilent model $5973 \mathrm{~N}$ MSD mass spectrometer (MS). The injector temperature was maintained at $250{ }^{\circ} \mathrm{C}$. The $\mathrm{GC}$ conditions in the GC-MS analysis were the same as in the $\mathrm{GC}$ analysis. The carrier gas (helium) flow rate was $1 \mathrm{~mL} / \mathrm{min}$. The electron energy was $70 \mathrm{eV}$ at $230^{\circ} \mathrm{C}$. The constituents were identified by matching their spectra with those recorded in a MS library (Wiley 7n). In addition, the constituents were confirmed by using the Kovats indices or GC retention time data with those of authentic standards or by publication literature.

(4) Statistical Analysis: The data were subjected to a mono-factorial variance analysis, with Duncan's multiple range method used by a significance of differences of $p<0.05$ (SPSS Base 12.0).

\section{Conclusions}

Thirty-five volatile components were identified in Narcissus flowers. The main aroma components for narcissus flowers were (Z)-ocimene, benzyl acetate, linalool, and indole. More ester compounds were identified in single-flowered samples than in double-flowered samples, which may contribute to the aroma profile of the flowers. The double-flowered samples had higher contents of 1,8-cineole than the single-flowered samples. During the vase life, it was found that the total volatile content peak area was greatest for single-flowered samples on day 2, while that for double-flowered samples occurred on day 3. The volatile constituents throughout the vase life of Narcissus tazetta var. chinensis Roem were reported for the first time in this study.

\section{Acknowledgments}

This work was supported by a research grant from China Medical University (CMU100-T-15 and CMU101-S-31), Taiwan.

\section{Conflicts of Interest}

The authors declare no conflict of interest.

\section{References}

1. Zou, Q.-C.; Zhuang, X.-Y.; Lu, G.; Jing, H.-F. Construction of expression plasmid vector containing the anti sense PSY gene and its transformation to Narcissus tazetta var. chinensis. J. Zhejiang For. Sci. Technol. 2006, 26, 25-30.

2. Fu, K.-L.; Shen, Y.-H.; Lu, L.; Li, B.; Yang, X.-W.; Su, J.; Liu, R.-H.; Zhang, W.-D. Two unusual rearranged flavan derivatives from Narcissus tazetta var. chinensis. Helv. Chim. Acta 2013, 96, 338-344. 
3. Ehret, C.; Maupetit, P.; Petrzilka, M. New organoleptically important components of Narcissus absolute (Narcissus poeticus L.). J. Essent. Oil Res. 1992, 4, 41-47.

4. Van Dort, H.M.; Jägers, P.P.; ter Heide, R.; van der Weerdt, A.J.A. Narcissus trevithian and Narcissus geranium. Analysis and Synthesis of Compounds. J. Agric. Food Chem. 1993, 41, 2063-2075.

5. Sakai, T.; Nishimura, K.; Hirose, Y. Narcissus tazetta var. chinensis. Nippon Kagaku Zasshi 1961, 82, 1716-1718.

6. Shikiev, A.S.; Goryaev, M.I.; Sharipova, F.S.; Serkerov, S.V. Chemical composition of the absolute oil of Narcissus tazetta florepleno. Khim. Prir. Soedin 1972, 3, 405-406.

7. Sakai, T. Study of essential oil constituents by capillary column GC/MS. Shitsuryo Bunseki 1979, 27, 202R-205R.

8. Joulain, D. Study of the fragrance given off by certain spring-time flowers. In Progress in Essent. Oil Research; Brunke, E.J., Ed.; Walter De Gruyter Incorporated: Berlin, Germany, 1986.

9. Loo, A.; Richard, H. A Study on Narcissus Absolute Composition. In Proceedings of the 10th Essential Oil Congress, Washington, DC, USA, 16-20 November 1986; p. 355.

10. Mookherjee, D.; Trenkle, R.W.; Wilson, R.A. Live vs. Dead Part II. A comparative analysis of the headspace volatiles of some important fragrance and flavor raw materials. J. Essent. Oil Res. 1989, 1, 85-90.

11. Dai, L.; Yang, L.; Guo, Y.; Peng, Q. Study on the chemical constituents of the essential oil of Zhangzhou narcissus flowers. Sерu 1990, 8, 377-380.

12. Zhu, L.F. New resources of essential oils in China. Perfum. Flavor. 1991, 16, 1-5.

13. Bruno, S.; de Laurentis, N.Y. Amico, A.; Stefanizzi, L. Chemical investigation and cytologic localization of essential oils in the flowers of Narcissus tazetta. Int. J. Pharmacog. 1994, 32, 357-361.

14. Arai, T. Volatile compounds of Narcissus taztta var. chinensis flowers. Koryo 1994, 184, 105-111.

15. Melliou, E.; Kalpoutzakis, E.; Tsitsa, E.; Magiatis, P. Composition of the Essential Oils of Narcissus tazetta and Narcissus serotinus from Greece. J. Essent. Oil Bearing Plants 2007, 10, 101-103.

16. Ferri, D.; Adami, M.; De Santis, A.; Ubaldi, C.; Cassaccia, E.C.; Oratore, A.; Fracassi, P.; Gioia, E. Traditional and supercritical $\mathrm{CO}_{2}$ extraction of the volatiles from Narcissus poeticus L. Perfum. Flavor. 2009, 34, 30-35.

17. Surburg, H.; Guentert, M.; Harder, H. Volatile compounds from flowers. Analytical and olfactory aspects. In Bioactive Volatile Compounds from Plants, ACS Symp. Series 525; Teranishi, R., Buttery, R.G., Sugisawa, H., Eds.; American Chemical Society: Washington, DC, USA, 1993, pp. 168-186.

18. Drew, D.P.; Rasmussen, S.K.; Avato, P.; Simonsen, H.T. A comparison of headspace solid-phase microextraction and classic hydrodistillation for the identification of volatile constituents from Thapsia spp. provides insights into guaianolide biosynthesis in Apiaceae. Phytochem. Anal. 2012, 23, 44-51.

19. Flamini, G.; Cioni, P.L.; Morelli, I. Differences in the fragrances of pollen, leaves, and floral parts of garland (Chrysanthemum coronarium) and composition of the essential oils from flowerheads and leaves. J. Agric. Food Chem. 2003, 51, 2267-2271. 
20. Li, Z.-G.; Lee, M.-R.; Shen, D.-L. Analysis of volatile compounds emitted from fresh Syringa oblata flowers in different florescence by headspace solid-phase microextraction-gas chromatography-mass spectrometry. Anal. Chim. Acta 2006, 576, 43-49.

21. Shang, C.-Q.; Hu, Y.-M.; Deng, C.-H.; Hu, K.-J. Rapid determination of volatile constituents of Michelia alba flowers by gas chromatography-mass spectrometry with solid-phase microextraction. J. Chromatogr. A 2002, 942, 283-288.

22. Rohloff, J. Monoterpene composition of essential oil from peppermint (Mentha $\times$ piperita L.) with regard to leaf position using solid-phase microextraction and gas chromatography/mass spectrometry analysis. J. Agric. Food Chem. 1999, 47, 3782-3786.

23. Peng, L.-W.; Sheu, M.-J.; Lin, L.Y.; Wu, C.-T.; Chiang, H.-M.; Lin, W.-H.; Lee, M.-C.; Chen, H.-C. Effect of Heat treatments on the Essential Oils of Kumquat (Fortunella margarita Swingle). Food Chem. 2013, 136, 532-537.

24. Chen, H.-C.; Sheu, M.-J.; Wu, C.-M. Characterization of volatiles in Guava (Psidium guajava L. cv. Chung-Shan-Yueh-Pa) Fruits from Taiwan. J. Food Drug Anal. 2006, 14, 398-402.

25. Oyama-Okubo, N.; Nakayama, M.; Ichimura, K. Control of floral scent emission by inhibitors of phenylalanine ammonia-lyase in cut flower of Lilium cv. 'Casa Blanca'. J. Japan. Soc. Hort. Sci. 2011, 80, 190-199.

26. Chen, H.-C.; Sheu, M-J.; Lin, L.-Y.; Wu, C.-M. Chemical composition of the leaf essential oil of Psidium guajava L. from Taiwan. J. Essent. Oil Res. 2007, 19, 345-347.

27. The Pherobase. Available online: http://www.pherobase.com (accessed on 5 November 2013).

28. Terpenoids Library List. Available online: http://massfinder.com/wiki/Terpenoids_Library_List (accessed on 5 November 2013).

29. Schomburg, G.; Dielmann, G. Identification by means of retention parameters. J. Chromatogr. Sci. 1973, 11, 151-159.

Sample Availability: Not available.

(C) 2013 by the authors; licensee MDPI, Basel, Switzerland. This article is an open access article distributed under the terms and conditions of the Creative Commons Attribution license (http://creativecommons.org/licenses/by/3.0/). 